\title{
Postoperative fluid collection in a patient with hydatid cyst of the liver
}

\author{
Yildirim $\mathrm{M}^{1 *}$, Adibelli $\mathrm{ZH}^{2}$ and Yazici $\mathrm{H}^{1}$ \\ ${ }^{1}$ Department of General Surgery, University of Health Sciences, Izmir Bozyaka Education and Research Hospital, Turkey \\ ${ }^{2}$ Department of Radiology, University of Health Sciences, Izmir Bozyaka Education and Research Hospital, Turkey
}

\section{Case report}

A 55-year-old woman patient was referred to our clinic with the diagnosis of hydatid cyst of the liver. In her CT a lesion measuring $15 \mathrm{~cm}$ in diameter was seen in the liver. At laparotomy, we found 15 $\mathrm{cm}$. cystic mass in liver segments III of the left lobe to VI of the right lobe. Cyts was found surrounded by stomach and transverse colon. We performed partial cystectomy and cyst evacuation (Figure 1 and 2). We could not make introflexion because of the non-elasticity of the hepatic parenchyma. We leaved a silastic tube to the cyst cavity. Histological examination showed hydatid membrane with multiple vesicular cysts. The postoperative period was uneventhful. Treatment was started with Albendazole in order to prevent recurrence of hydatid disease. An abdomen CT evaluation at the 6th months showed postoperative changes and fluid collection $15 \mathrm{~cm}$ in diameter localized to the cystotomy side (Figure 3). After evaluation and informed consent we performed an ultrasound-guided drainage resulted in collection of about $200 \mathrm{~mL}$ of a yellowish aspirate from the cyst location. On the 6 th day after the drainage the ultrasound showed a lesion shrinkage to $3 \mathrm{~cm}$. She is free of recurrence or fluid collection 18 months after drainage.

Echinococcal hydatid cysts of the liver is frequently seen in endemic regions [1]. The treatment of hydatid cyst is surgical. Desired goals of treatment of liver hydatidosis are complete elimination of parasite and prevention of recurrent disease [2-4]. Pericystectomy is presently considered to be ideal operation. As a general rule, prompt emptying of the cyst must be performed to prevent risk of cyst perforation. If the pericyst has been removed and hemorhage or bile leakage excluded, then a omental flab can be used on residual cavity. Once the residual cyst wall has been controlled, introflexion can be performed to prevent a dead space. In spite of this procedures, late follow-up image studies may show fluid collections usually as bilomas and hematomas. Most important problem is to decide either a reccurent cyst or the remnant of previous surgery [5]. The pattern of postoperative symptoms usually includes a sensation of pressure and deep-seated pain at the upper abdominal quadrant. CT is valuable for the diagnosis of recurring patterns. It might be difficult to decide differantial diagnosis of fluid collections. The treatment methods are varied.

\section{Conclusion}

In cases of manifest collections, ultrasonography-guided emptying of huge collections is useful to relieve the clinic symptoms. With this case we woud like to share our experience with this postoperative follow-up and drainage procedure without residual collections.

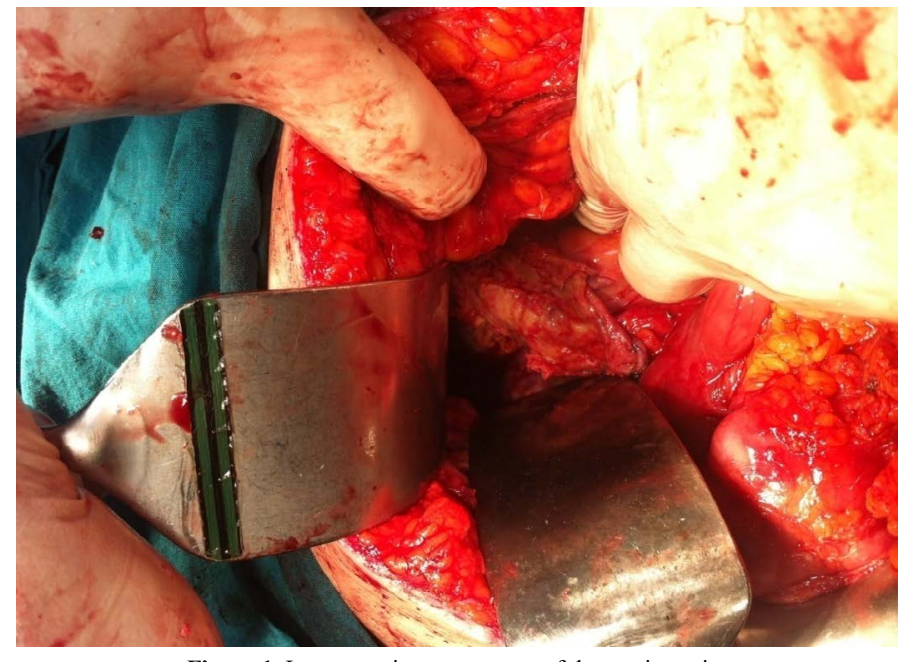

Figure 1. Intraoperative appearance of the cystic cavity

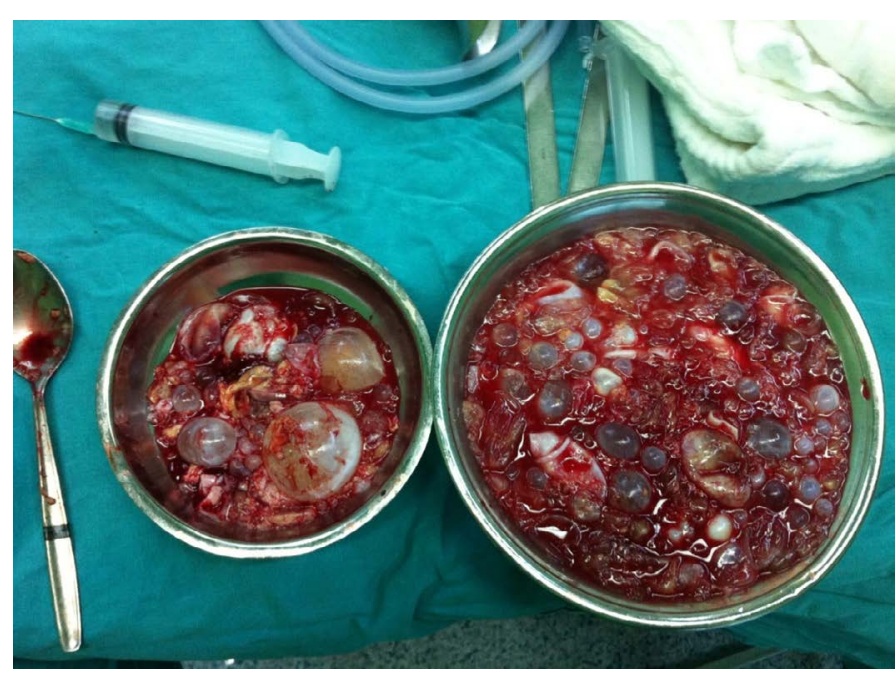

Figure 2. Macroscopic view of the vesicles

Correspondence to: Mehmet Yildirim, Department of General Surgery, University of Health Sciences, Izmir Bozyaka Education and Research Hospital, Turkey, E-mail: m.yildirm@sbu.edu.tr

Received: November 28, 2017; Accepted: December 15, 2017; Published: December 19, 2017 


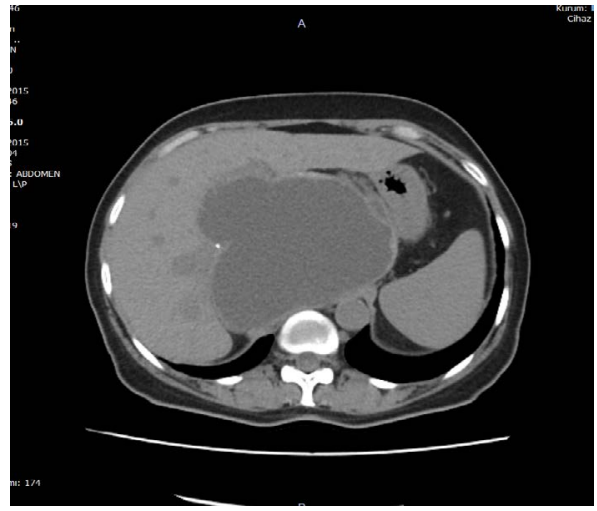

\section{References}

1. Pakala T, Molina M, Wu GY (2016) Hepatic echinococcal cysts: A review. J Clin Trans Hepatol 4: 39-46.

2. Sozuer E, Akyuz M, Akbulut S (2014) Open surgery for hydatid disease. Int Surg 99: 764-769.

3. Buttenschoen K, Carli Buttenschoen D (2003) Echinococcus granulosus infection: the challenge of surgical treatment. Langenbecks Arch Surg 388: 218-230.

4. 4.Tuxun T, Zhang JH, Zhao JM, Tai QW, Abudurexti M, et al. (2014) World review of laparoscopic treatment of liver cystic echinococcosis-914 patients. Int J Infect Dis 24: $43-50$.

5. 5.Yildirim M, Engin O, Oztekin O, Akdamar F, Adibelli ZH, et al. (2009) Diagnostic evaluation and surgical management of recurrent hydatid cyst in an endemic region. Radiol Oncol 43: 162-169

Figure 3. CT scan showed a large fluid collection in the right lobe of the liver.

Copyright: (C2017 Yildirim M. This is an open-access article distributed under the terms of the Creative Commons Attribution License, which permits unrestricted use, distribution, and reproduction in any medium, provided the original author and source are credited. 